\title{
Can the subterranean fauna be used as proxy for past environmental changes? - the example of the Carpathians cave fauna
}

\author{
Oana Teodora Moldovan ${ }^{\ddagger}$, Ionut Cornel Mirea§, Marius Kenesz ${ }^{\ddagger}$ \\ ‡ Emil Racovitza Institute of Speleology, Cluj-Napoca, Romania \\ $\S$ Emil Racovitza Institute of Speleology, Bucharest, Romania
}

Corresponding author: Oana Teodora Moldovan (oanamol35@gmail.com)

Received: 31 Aug 2018 | Published: 04 Sep 2018

Citation: Moldovan O, Mirea I, Kenesz M (2018) Can the subterranean fauna be used as proxy for past environmental changes? - the example of the Carpathians cave fauna. ARPHA Conference Abstracts 1: e29437. https://doi.org/10.3897/aca.1.e29437

\section{Abstract}

Carpathian Mountains were one of the main refuge areas during the climate changes of the Pleistocene and the Holocene in Europe and one of the richest regions in the world in subterranean (caves and associated habitats) endemic species. Nevertheless, the Carpathian Mountains subterranean fauna importance is underestimated especially due to dispersed information on its diversity and the scarcity of molecular studies in the area. Here, we present a first general view of the cave fauna hotspot represented by the Romanian Carpathians and the geological and historical processes that shaped the patterns of subterranean distribution and diversity at regional scale. The Carpathians are an amalgam of various geological units with complex paleogeographical evolution that is reflected in completely different species assemblages dominated by unit specific fauna groups. Phylogeography of Coleoptera and environmental parameters are adding to the general view at regional scale and offer additional explanation for this exceptional subterranean diversification in a non-Mediterranean region. We also use the example of the Carpathians cave fauna as proxy for past environmental changes in the area. Troglobionts are endemic on small areas and by studying their present distributions and phylogeny, past processes of landscape evolution on the surface can be better understood. 


\section{Presenting author}

Oana Teodora Moldovan

\section{Presented at}

25th International Conference on Subterranean Biology 